Pacific Journal of Mathematics

ON COMPLETE SECOND ORDER LINEAR DIFFERENTIA

baNaCh SPAC 


\title{
ON COMPLETE SECOND ORDER LINEAR DIFFERENTIAL EQUATIONS IN BANACH SPACES
}

\author{
Xio TiJun and Liang Jin
}

\begin{abstract}
This paper is concerned with the complete second order equation $u^{\prime \prime}(t)+B u^{\prime}(t)+A u(t)=0$ in a Banach space, where both $A$ and $B$ are densely defined closed linear operators. The main result is a theorem of Hill-Yosida-Phillips type for the Cauchy problem for the equation to be well posed.
\end{abstract}

1. Introduction and the main result. Consider the complete second order linear differential equation

$$
u^{\prime \prime}(t)+B u^{\prime}(t)+A u(t)=0 \quad(t \geq 0)
$$

in a complete Banach space $E$, where $A, B$ are densely defined closed linear operators. The equation has been extensively studied by semigroup methods during the last thirty years. A great amount of literature on it can be looked up in Fattorini's monograph [1] which was published in 1985. However, as stated in [1, Ch. VIII], the theory of (1.1) "can hardly be said in definitive form".

Let us begin with the restatements of some definitions in [1]:

Definition 1. We say that an $E$-valued function $u(t)$ defined in $t \geq 0$ is a solution of (1.1) if $u(t)$ is twice continuously differentiable, $u(t) \in D(A), u^{\prime}(t) \in D(B), A u(t)$ and $B u^{\prime}(t)$ are continuous and (1.1) is satisfied in $t \geq 0$.

Definition 2. We say that the Cauchy problem for (1.1) is well posed if the following two assumptions are satisfied:

(a) There exist dense subspaces $D_{0}, D_{1}$ of $E$ such that, for any $u_{0} \in D_{0}, u_{1} \in D_{1}$, there exists a solution $u(t)$ of $(1.1)$ with $u(0)=u_{0}$, $u^{\prime}(0)=u_{1}$.

(b) There exists a nondecreasing, nonnegative function $N(t)$ defined in $t \geq 0$ such that

$$
\|u(t)\| \leq N(t)\left(\|u(0)\|+\left\|u^{\prime}(0)\right\|\right) \quad(t \geq 0)
$$

for any solution of (1.1). 
Our definition of well posed Cauchy problem corresponds to that of uniformly well posed Cauchy problem in [1]. Thus, in quoting results from [1], "uniformly well posed" should be substituted by "well posed". See also [2].

Definition 3. Assume that the Cauchy problem for (1.1) is well posed. Define, for $t \geq 0, u \in D_{0}, v \in D_{1}$,

$$
C(t) u=u(t), \quad S(t) v=v(t),
$$

where $u(t)$ (resp. $v(t)$ ) is the solution of $(1.1)$ with $u(0)=u, u^{\prime}(0)=0$ (resp. $v(0)=0, v^{\prime}(0)=v$ ). In view of $(1.2), C(t)$ (resp. $\left.S(t)\right)$ is a bounded operator in $D_{0}$ (resp. $\left.D_{1}\right)$. Since $D_{0}$ (resp. $\left.D_{1}\right)$ is dense in $E$ we can extend $C(t)$ (resp. $S(t)$ ) to a bounded operator on $E$, which we denote by the same symbol. We call the operator-valued functions $C(t)$ and $S(t)$ the propagators of (1.1).

If $B=0,(1.1)$ becomes the incomplete equation

$$
u^{\prime \prime}(t)+A u(t)=0 \quad(t \geq 0) .
$$

According to $[1,8,9]$, if the Cauchy problem for (1.3) is well posed then the solutions grow exponentially and a phase space exists; the well posedness is completely determined by the resolvent of $A$, that is

TheOREM A [1, 8, 9]. The Cauchy problem for (1.3) is well posed if and only if there exist constants $C, \omega \geq 0$ such that for $\operatorname{Re} \lambda>\omega$, $\left(\lambda^{2} I+A\right)^{-1} \in L(E)$ (the set of bounded linear operators on $E$ ) and

$$
\left\|\left[\lambda\left(\lambda^{2} I+A\right)^{-1}\right]^{(n)}\right\| \leq C n !(\operatorname{Re} \lambda-\omega)^{-n-1} \quad(n=0,1,2, \ldots) .
$$

However, for the complete equation (1.1), many problems are difficult to discuss if we use the same definition of well posed problem. We may encounter paradoxical situations entailing loss of exponential growth of solutions and nonexistence of phase spaces as has been illustrated by Fattorini [1] with a counterexample. For this, Fattorini has introduced the following

Assumption 3.1 [1, Ch. VIII]. (a) $S(t) u$ is continuously differentiable in $t \geq 0$ for all $u \in E$.

(b) $S(t) E \subseteq D(B)$ and $B S(t)$ is continuous in $t \geq 0$ for all $u \in E$. And he has shown that Assumption 3.1 guarantees exponential growth of solutions and existence of a state space. 
Definition 4. We say that the Cauchy problem for (1.1) is strongly well posed it is well posed and Assumption 3.1 is satisfied.

When $B=0$, strong well posedness is equivalent to well posedness.

The problem arises of giving necessary and sufficient conditions on $A$ and $B$ for strong well posedness of the Cauchy problem. We give a solution to this problem by proving

THEOREM 1. For equation (1.1) the following statements are equivalent:

(i) The Cauchy problem for (1.1) is strongly well posed.

(ii) There exists a complex number $\lambda_{0}$ such that $\Delta\left(\lambda_{0}\right)=\lambda_{0}^{2} I+\lambda_{0} B+$ $A$ is closed and densely defined, and $\Delta\left(\lambda_{0}\right)\left(D\left(\Delta\left(\lambda_{0}\right)\right)\right)=E$. The Cauchy problem for (1.1) is well posed and (1.1) has a solution for every initial value $\left(u_{0}, u_{1}\right) \in(D(A) \cap D(B))^{2}=(D(A) \cap D(B)) \times(D(A) \cap D(B))$.

(iii) $D(A) \cap D(B)$ is dense in $E$. There exist constants $C, \omega \geq 0$ such that for $\operatorname{Re} \lambda>\omega, \Delta(\lambda)^{-1}=\left(\lambda^{2} I+\lambda B+A\right)^{-1} \in L(E), \Delta(\lambda)^{-1} A$ is closable and

$$
\begin{aligned}
\left\|\left[\lambda \Delta(\lambda)^{-1}\right]^{(n)}\right\| & \leq C n !(\operatorname{Re} \lambda-\omega)^{-n-1} \quad(n=0,1,2, \ldots), \\
\left\|\left[B \Delta(\lambda)^{-1}\right]^{(n)}\right\| & \leq C n !(\operatorname{Re} \lambda-\omega)^{-n-1} \quad(n=0,1,2, \ldots), \\
\left\|\left[\Delta(\lambda)^{-1} B u\right]^{(n)}\right\| & \leq C n !(\operatorname{Re} \lambda-\omega)^{-n-1}\|u\|
\end{aligned}
$$

$$
(u \in D(A) \cap D(B), n=0,1,2, \ldots) .
$$

Moreover, if (iii) is satisfied, we have three kinds of explicit expressions for the propagators:

$$
C(t)=\left.\operatorname{Lim}_{n \rightarrow \infty} \frac{(-1)^{n}}{n !}\left(\frac{n}{t}\right)^{n+1}\left[\frac{1}{\lambda} I-\frac{1}{\lambda} \overline{\Delta(\lambda)^{-1} A}\right]^{(n)}\right|_{\lambda=n / t}
$$

$$
\begin{aligned}
& S(t)=\left.\operatorname{Lim}_{n \rightarrow \infty} \frac{(-1)^{n}}{n !}\left(\frac{n}{t}\right)^{n+1}\left[\Delta(\lambda)^{-1}\right]^{(n)}\right|_{\lambda=n / t} \quad(t>0) ; \\
& C(t) u=u-\operatorname{Lim}_{\lambda \rightarrow \infty} \sum_{n=1}^{\infty}(-1)^{n+1} \frac{1}{n !} e^{n \lambda t} \Delta(n \lambda)^{-1} A u \\
& \quad(u \in D(A), t \geq 0),
\end{aligned}
$$

(1.7) $S(t) u=\operatorname{Lim}_{\lambda \rightarrow \infty} \lambda \sum_{n=1}^{\infty}(-1)^{n-1} \frac{1}{(n-1) !} e^{n \lambda t} \Delta(n \lambda)^{-1} u$

$(u \in E, t \geq 0)$ 
and for $\nu>\omega$,

$$
\begin{aligned}
& C(t) u=u-\frac{1}{2 \pi i} \int_{\nu-i \infty}^{\nu+i \infty} \frac{1}{\lambda} e^{\lambda t} \Delta(\lambda)^{-1} A u d \lambda \\
& \quad(u \in D(A), t \geq 0), \\
& S(t) u=\frac{1}{2 \pi i} \int_{\nu-i \infty}^{\nu+i \infty} e^{\lambda t} \Delta(\lambda)^{-1} u d \lambda \quad(u \in E, t \geq 0) .
\end{aligned}
$$

REMARK 1. Theorem 1 contains Theorem A.

REMARK 2. Although the implication (iii) $\Rightarrow$ (i) could be possibly proved by direct application of the Hill-Yosida theorem, complications stemming from lack of commutativity of $A$ and $B$ make this approach impractical.

2. Proof of Theorem 1. First of all, we present three lemmas.

LEMMA 1. Let $f(t)$ be an E-valued continuous function defined in $t \geq 0$ such that $\int_{0}^{\infty} e^{-c t} f(t) d t$ exists for some positive $c$. Then, as $k \rightarrow \infty$,

$$
\begin{aligned}
& M_{k}(t)=\left(\frac{k}{t}\right)^{k} \frac{1}{(k-1) !} \int_{0}^{\infty} e^{-k s / t} s^{k-1} f(s) d s \rightarrow f(t), \\
& N_{k}(t)=\left(\frac{k}{t}\right)^{k+1} \frac{1}{k !} \int_{0}^{\infty} e^{-k s / t} s^{k} f(s) d s \rightarrow f(t),
\end{aligned}
$$

uniformly on compact subsets of $t>0$.

The proof of (2.2) is essentially the same as the one of [11, P. 285, Th. 5a] and we omit it. (2.1) follows immediately from $M_{k}(t)=$ $N_{k-1}((k-1) t / k)$.

LEMMA 2. Let $f(t)$ be an E-valued continuous function with $\|f(t)\| \leq$ $C e^{\omega t}$ in $t \geq 0$, where $C, \omega \geq 0$, then

$$
\int_{0}^{t} f(s) d s=\operatorname{Lim}_{\lambda \rightarrow \infty} \sum_{n=1}^{\infty} \frac{(-1)^{n-1}}{n !} e^{n \lambda t} \int_{0}^{\infty} e^{-n \lambda r} f(r) d r \quad(t \geq 0) .
$$

The proof is completely the same as the first part in the proof of (Phragmén's representation theorem, see [6]) and we also omit it. 
LEMMA 3. Let $f(t)$ be an E-valued continuously differentiable function with $\|f(t)\| \leq C e^{\omega t}$ in $t \geq 0$, where $C, \omega \geq 0$, then for $\bar{\omega}>\omega$

$$
f(t)=\frac{1}{2 \pi i} \int_{\bar{\omega}-i \infty}^{\bar{\omega}+i \infty} e^{\lambda t}\left[\int_{0}^{\infty} e^{-\lambda s} f(s) d s\right] d \lambda \quad(t>0) .
$$

Proof. By [4, Th. 6.3.1],

$$
f(t)=\operatorname{Lim}_{T \rightarrow \infty} \frac{1}{2 \pi i} \int_{\bar{\omega}-i T}^{\bar{\omega}+i T} e^{\lambda t}\left[\int_{0}^{\infty} e^{-\lambda s} f(s) d s\right] d \lambda \quad(t>0) .
$$

It remains to show that the integral

$$
\int_{\bar{\omega}+i \infty}^{\bar{\omega}+i \infty} e^{\lambda t}\left[\int_{0}^{\infty} e^{-\lambda s} f(s) d s\right] d \lambda
$$

converges. We can prove this fact using arguments similar to those of [11, P. 68, Th. 7.5], noting that Riemann-Lebesgue theorem is applicable to vector-valued functions (see [3, P. 401]) and making use of the estimate $\|f(t)\| \leq C e^{\omega t}$ for $t \geq 0$.

Proof of Theorem 1. (i) $\Rightarrow$ (ii). By [1, §VIII.3].

(ii) $\Rightarrow\left(\right.$ i). Let $u(t)$ be a solution of (1.1) with $u(0), u^{\prime}(0) \in D(A) \cap$ $D(B)$, then $u(t) \in D(A)$ for $t \geq 0, B u^{\prime}(t)$ is continuous in $t \geq 0$ and therefore $B u^{\prime}(t)$ is integrable on any bounded interval of $t \geq 0$. Hence $u(t)=u(0)+\int_{0}^{t} u^{\prime}(s) d s \in D(B)$ for $t \geq 0$. Set $v(t)=e^{-\lambda_{0} t} u(t)$; then

$$
\begin{aligned}
u^{\prime}(t) & =\lambda_{0} e^{\lambda_{0} t} v(t)+e^{\lambda_{0} t} v^{\prime}(t), \\
u^{\prime \prime}(t) & =\lambda_{0}^{2} e^{\lambda_{0} t} v(t)+2 \lambda_{0} e^{\lambda_{0} t} v^{\prime}(t)+e^{\lambda_{0} t} v^{\prime \prime}(t)
\end{aligned}
$$

Since $v(t) \in D(A) \cap D(B)$, we have

$$
v^{\prime \prime}(t)+B_{1} v^{\prime}(t)+A_{1} v(t)=0
$$

where $B_{1}=B+2 \lambda_{0} I, A_{1}=\Delta\left(\lambda_{0}\right)=\lambda_{0}^{2} I+\lambda_{0} B+A$. Obviously $u(0)=$ $v(0), u^{\prime}(0)=\lambda_{0} v(0)+v^{\prime}(0)$; hence for every initial value $\left(v(0), v^{\prime}(0)\right) \in$ $\left(D\left(A_{1}\right)\right)^{2}=(D(A) \cap D(B))^{2}$ the equation (2.3) has a solution. It is easily verified that if $v(t)$ is a solution of (2.3) then $u(t)=e^{\lambda_{0} t} v(t)$ is a solution of (1.1). From these observations we deduce that the Cauchy problem for (2.3) is well-posed. Denoting the propagators of (2.3) by $C_{1}(t)$ and $S_{1}(t)$, clearly $S_{1}(t)=e^{-\lambda_{0} t} S(t)$ for $t \geq 0$. Since $A_{1}\left(D\left(A_{1}\right)\right)=E$. Assumption 3.1 is then satisfied for equation (2.3) in view of [2, Th. 4.1(b)]. Thus Assumption 3.1 holds for equation (1.1) and this completes the proof. 
(i) $\Rightarrow$ (iii). According to [1], there exist constants $C, \omega \geq 0$ such that for $\operatorname{Re} \lambda>\omega, \Delta(\lambda)^{-1} \in L(E)$ and

$$
\begin{array}{ll}
\Delta(\lambda)^{-1} u=\int_{0}^{\infty} e^{-\lambda t} S(t) u d t & (u \in E) \\
u-\int_{0}^{t} S(s) A u d s=C(t) u & (t \geq 0, u \in D(A))
\end{array}
$$

and for $t \geq 0$.

$$
\left\|S^{\prime}(t)\right\| \leq C e^{\omega t}, \quad\|B S(t)\| \leq C e^{\omega t}, \quad\|C(t)\| \leq C e^{\omega t} .
$$

Consequently, for $u \in E$,

$$
\begin{aligned}
& \lambda \Delta(\lambda)^{-1} u=\int_{0}^{\infty} \lambda e^{-\lambda t} S(t) u d t=\int_{0}^{\infty} e^{-\lambda t} S^{\prime}(t) u d t \\
& B \Delta(\lambda)^{-1} u=\int_{0}^{\infty} e^{-\lambda t} B S(t) u d t
\end{aligned}
$$

Hence

$$
\begin{aligned}
\left\|\left[\lambda \Delta(\lambda)^{-1}\right]^{(n)}\right\| & \leq \int_{0}^{\infty} t^{n} e^{-\operatorname{Re} \lambda t} C e^{\omega t} d t \\
& =C n !(\operatorname{Re} \lambda-\omega)^{-n-1} \quad(n=0,1,2, \ldots), \\
\left\|\left[B \Delta(\lambda)^{-1}\right]^{(n)}\right\| & \leq \int_{0}^{\infty} t^{n} e^{-\operatorname{Re} \lambda t} C e^{\omega t} d t \\
& =C n !(\operatorname{Re} \lambda-\omega)^{-n-1} \quad(n=0,1,2, \ldots) .
\end{aligned}
$$

Also

$$
\begin{aligned}
\frac{1}{\lambda} \Delta(\lambda)^{-1} A u & =\frac{1}{\lambda} \int_{0}^{\infty} e^{-\lambda t} S(t) A u d t \\
& =\int_{0}^{\infty} e^{-\lambda t}\left[\int_{0}^{t} S(s) A u d s\right] d t \quad(u \in D(A))
\end{aligned}
$$

Then for $u \in D(A), n=0,1,2, \ldots$,

$$
\begin{aligned}
\left\|\left[\frac{1}{\lambda} \Delta(\lambda)^{-1} A u\right]^{(n)}\right\| & \leq \int_{0}^{\infty} t^{n} e^{-\operatorname{Re} \lambda t}\left(1+C e^{\omega t}\right)\|u\| d t \\
& \leq(C+1) n !(\operatorname{Re} \lambda-\omega)^{-n-1}\|u\| .
\end{aligned}
$$

But

$$
\Delta(\lambda)^{-1} B u=\frac{1}{\lambda} u-\lambda \Delta(\lambda)^{-1} u-\frac{1}{\lambda} \Delta(\lambda)^{-1} A u \quad(u \in D(A) \cap D(B)) .
$$


Thus, we obtain

$$
\begin{array}{r}
\left\|\left[\Delta(\lambda)^{-1} B u\right]^{(n)}\right\| \leq 2(C+1) n !(\operatorname{Re} \lambda-\omega)^{-n-1}\|u\| \\
(u \in D(A) \cap D(B), n=0,1,2, \ldots) .
\end{array}
$$

This ends the proof of the implication (i) $\Rightarrow$ (iii).

(iii) $\Rightarrow\left(\right.$ i). We define a linear operator $G=\left(\begin{array}{cc}0 & -I \\ A & B\end{array}\right)$ in the space $E \times E$ with domain $D(G)=D(A) \times(D(A) \cap D(B))$. It is easy to verify that for $\lambda>\omega$,

$$
\begin{aligned}
(\lambda I+G)^{-1} & =\left(\begin{array}{cc}
\frac{1}{\lambda} I-\Delta(\lambda)^{-1} A & \Delta(\lambda)^{-1} \\
-\Delta(\lambda)^{-1} A & \lambda \Delta(\lambda)^{-1}
\end{array}\right), \\
D\left((\lambda I+G)^{-1}\right) & =D(A) \times E .
\end{aligned}
$$

By virtue of the equality $\frac{1}{\lambda} \Delta(\lambda)^{-1} A u=\frac{1}{\lambda} u-\lambda \Delta(\lambda)^{-1} u-\Delta(\lambda)^{-1} B u$ for $u \in D(A) \cap D(B)$ and the fact that $\Delta(\lambda)^{-1} A$ is closable, we can extend $\Delta(\lambda)^{-1} A$ to a bounded operator on $E$ and therefore can extend $(\lambda I+G)^{-1}$ to a bounded operator on $E \times E$, which is $\overline{(\lambda I+G)^{-1}}$. Accordingly, $G$ is closable and $(\lambda I+\bar{G})^{-1}=\overline{(\lambda I+G)^{-1}}$. By [10, P. 73], for $t>0, n>\omega t$,

$$
\frac{d}{d t}\left[\left(\frac{n}{t}\right)^{n}\left(\frac{n}{t} I+\bar{G}\right)^{-n}\right]=-\left(\frac{n}{t}\right)^{n+1}\left(\frac{n}{t} I+\bar{G}\right)^{-n} \bar{G}\left(\frac{n}{t} I+\bar{G}\right)^{-1} .
$$

Set, for $u \in D(A), v \in E$,

$$
\begin{aligned}
X_{n}(t ; u, v)= & {\left[\left(\frac{n}{t}\right)^{n}\left(\frac{n}{t} I+G\right)^{-n}\right]\left(\begin{array}{c}
u \\
v
\end{array}\right), } \\
U_{n}(t ; u, v)= & \frac{(-1)^{n-1}}{(n-1) !}\left(\frac{n}{t}\right)^{n}\left\{\left[\frac{1}{\lambda} u-\frac{1}{\lambda} \Delta(\lambda)^{-1} A u\right]^{(n-1)}\right. \\
& \left.+\left[\Delta(\lambda)^{-1} v\right]^{(n-1)}\right\}\left.\right|_{\lambda=n / t}, \\
V_{n}(t ; u, v)= & \frac{(-1)^{n-1}}{(n-1) !}\left(\frac{n}{t}\right)^{n}\left\{\left[-\Delta(\lambda)^{-1} A u\right]^{(n-1)}\right. \\
& \left.+\left[\lambda \Delta(\lambda)^{-1} v\right]^{(n-1)}\right\}\left.\right|_{\lambda=n / t}
\end{aligned}
$$

Then obviously

$$
\frac{d}{d t} X_{n}(t ; u, v)+G X_{n+1}\left(\frac{n+1}{n} t ; u, v\right)=0 .
$$


But

$$
\begin{aligned}
& X_{n}(t ; u, v)=\left\{\left.\frac{(-1)^{n-1}}{(n-1) !}\left(\frac{n}{t}\right)^{n}\left[(\lambda I+G)^{-1}\right]^{(n-1)}\right|_{\lambda=n / t}\right\}\left(\begin{array}{c}
u \\
v
\end{array}\right) \\
& =\left.\frac{(-1)^{n-1}}{(n-1) !}\left(\frac{n}{t}\right)^{n}\left(\begin{array}{c}
{\left[\frac{1}{\lambda} u-\frac{1}{\lambda} \Delta(\lambda)^{-1} A u\right]^{(n-1)}+\left[\Delta(\lambda)^{-1} v\right]^{(n-1)}} \\
{\left[-\Delta(\lambda)^{-1} A u\right]^{(n-1)}+\left[\lambda \Delta(\lambda)^{-1} v\right]^{(n-1)}}
\end{array}\right)\right|_{\lambda=n / t} \\
& =\left(\begin{array}{c}
U_{n}(t ; u, v) \\
V_{n}(t ; u, v)
\end{array}\right),
\end{aligned}
$$

hence we have

$$
\left\{\begin{array}{l}
\frac{d}{d t} U_{n}(t ; u, v)=V_{n+1}\left(\frac{n+1}{n} t ; u, v\right) \\
\frac{d}{d t} V_{n}(t ; u, v)=-B V_{n+1}\left(\frac{n+1}{n} t ; u, v\right)-A U_{n+1}\left(\frac{n+1}{n} t ; u, v\right) .
\end{array}\right.
$$

Next, we shall discuss the convergence of (2.4) as $n \rightarrow \infty$. Define, for $t \geq 0, v \in D(A) \cap D(B)$,

$$
\begin{aligned}
\widetilde{S}(t) v= & t v-\frac{1}{2 \pi i} \int_{\bar{\omega}-i \infty}^{\bar{\omega}+i \infty} \frac{e^{\lambda t}}{\lambda^{2}}\left(\lambda \Delta(\lambda)^{-1}\right) B v d \lambda \\
& -\frac{1}{2 \pi i} \int_{\bar{\omega}-i \infty}^{\bar{\omega}+i \infty} \frac{e^{\lambda t}}{\lambda^{2}} \Delta(\lambda)^{-1} A v d \lambda \\
= & \frac{1}{2 \pi i} \int_{\bar{\omega}-i \infty}^{\bar{\omega}+i \infty} e^{\lambda t} \Delta(\lambda)^{-1} v d \lambda,
\end{aligned}
$$

where $\bar{\omega}>\omega$. Clearly $\widetilde{S}(0)=v=0, \widetilde{S}(t) v$ is continuous in $t \geq 0$ and for $t \geq 0$

$$
\begin{aligned}
\int_{0}^{t} \tilde{S}(s) v d s= & \frac{1}{2 \pi i} \int_{\bar{\omega}-i \infty}^{\bar{\omega}+i \infty} \frac{e^{\lambda t}}{\lambda^{2}} \lambda \Delta(\lambda)^{-1} v d \lambda, \\
B \int_{0}^{t} \tilde{S}(s) v d s= & \frac{1}{2 \pi i} \int_{\bar{\omega}-i \infty}^{\bar{\omega}+i \infty} \frac{e^{\lambda t}}{\lambda} B \Delta(\lambda)^{-1} v d \lambda \\
= & \frac{1}{2} t^{2} B v-\frac{1}{2 \pi i} \int_{\bar{\omega}-i \infty}^{\bar{\omega}+i \infty} \frac{e^{\lambda t}}{\lambda^{2}} B \Delta(\lambda)^{-1} B v d \lambda \\
& -\frac{1}{2 \pi i} \int_{\bar{\omega}-i \infty}^{\bar{\omega}+i \infty} \frac{e^{\lambda t}}{\lambda^{3}} B \Delta(\lambda)^{-1} A v d \lambda, \\
B \int_{0}^{t}(t-s) \widetilde{S}(s) v d s= & \frac{1}{2 \pi i} \int_{\bar{\omega}-i \infty}^{\bar{\omega}+i \infty} \frac{e^{\lambda t}}{\lambda^{2}} B \Delta(\lambda)^{-1} v d \lambda .
\end{aligned}
$$


Making use of the Fubini theorem and the Cauchy formula, we obtain that for $\mu>\bar{\omega}, k=0,1,2, \ldots$,

$$
\begin{aligned}
& \int_{0}^{\infty} e^{-\mu t}(-t)^{k} \tilde{S}(t) v d t \int_{0}^{\infty} e^{-\mu t}(-t)^{k}\left[\frac{1}{2 \pi i} \int_{\bar{\omega}-i \infty}^{\bar{\omega}+i \infty} e^{\lambda t} \Delta(\lambda)^{-1} v d \lambda\right] d t \\
&= \frac{(-1)^{k}}{2 \pi i} \int_{\bar{\omega}-i \infty}^{\bar{\omega}+i \infty} \Delta(\lambda)^{-1} v\left[\int_{0}^{\infty} e^{(\lambda-\mu) t} t^{k} d t\right] d \lambda=\left[\Delta(\mu)^{-1} v\right]^{(k)}, \\
& \int_{0}^{\infty} e^{-\mu t}(-t)^{k}\left[\int_{0}^{t} \tilde{S}(s) v d s\right] d t=\left[\frac{1}{\mu} \Delta(\mu)^{-1} v\right]^{(k)}, \\
& \int_{0}^{\infty} e^{-\mu t}(-t)^{k}\left[B \int_{0}^{t} \tilde{S}(s) v d s\right] d t=\left[\frac{1}{\mu} B \Delta(\mu)^{-1} v\right]^{(k)}, \\
& \int_{0}^{\infty} e^{-\mu t}(-t)^{k}\left[B \int_{0}^{t}(t-s) \widetilde{S}(s) v d s\right] d t=\left[\frac{1}{\mu^{2}} B \Delta(\mu)^{-1} v\right]^{(k)} .
\end{aligned}
$$

This and the obvious fact that $\widetilde{S}(t) v, \int_{0}^{t} \widetilde{S}(s) v d s, B \int_{0}^{t} \widetilde{S}(s) v d s$ and $B \int_{0}^{t}(t-s) \widetilde{S}(s) v d s$ are continuous in $t \geq 0$, in view of (2.1) in Lemma 1 , show that as $n \rightarrow \infty$

$$
\left.\frac{(-1)^{n-1}}{(n-1) !}\left(\frac{n}{t}\right)^{n}\left[\Delta(\lambda)^{-1} v\right]^{(n-1)}\right|_{\lambda=n / t} \rightarrow \widetilde{S}(t) v \quad(t>0),
$$

$$
\left.\frac{(-1)^{n-1}}{(n-1) !}\left(\frac{n}{t}\right)^{n}\left[\frac{1}{\lambda} \Delta(\lambda)^{-1} v\right]^{(n-1)}\right|_{\lambda=n / t} \rightarrow \int_{0}^{t} \tilde{S}(s) v d s
$$

$$
\left.\frac{(-1)^{n-1}}{(n-1) !}\left(\frac{n}{t}\right)^{n}\left[\frac{1}{\lambda} B \Delta(\lambda)^{-1} v\right]^{(n-1)}\right|_{\lambda=n / t} \rightarrow B \int_{0}^{t} \tilde{S}(s) v d s
$$

(2.8) $\left.\frac{(-1)^{n-1}}{(n-1) !}\left(\frac{n}{t}\right)^{n}\left[\frac{1}{\lambda^{2}} B \Delta(\lambda)^{-1} v\right]^{(n-1)}\right|_{\lambda=n / t} \rightarrow B \int_{0}^{t}(t-s) \widetilde{S}(s) v d s$

uniformly on compact subsets of $t>0$. It is easy to verify by the Leibniz formula that $\left\|\left[\Delta(\lambda)^{-1}\right]^{(n)}\right\|,\left\|\left[\frac{1}{\lambda} \Delta(\lambda)^{-1}\right]^{(n)}\right\|$ and $\left\|\left[\frac{1}{\lambda^{2}} B \Delta(\lambda)^{-1}\right]^{(n)}\right\|$ are all bounded by $C n !(\operatorname{Re} \lambda-\omega)^{-n-1}$ for $\operatorname{Re} \lambda>\omega, n=0,1,2, \ldots$ (if the 
constant is still denoted by $C)$. Therefore for $t>0, v \in D(A) \cap D(B)$,

$$
\begin{aligned}
& \|\widetilde{S}(t) v\|=\left\|\left.\operatorname{Lim}_{n \rightarrow \infty} \frac{(-1)^{n-1}}{(n-1) !}\left(\frac{n}{t}\right)^{n}\left[\Delta(\lambda)^{-1} v\right]^{(n-1)}\right|_{\lambda=n / t}\right\| \\
& \leq \operatorname{Lim}_{n \rightarrow \infty} \frac{C}{(n-1) !}\left(\frac{n}{t}\right)^{n}(n-1) !\left(\frac{n}{t}-\omega\right)^{-n}\|v\| \\
& =\operatorname{Lim}_{n \rightarrow \infty} C\left(1-\frac{\omega t}{n}\right)^{-n}\|v\|=C e^{\omega t}\|v\|, \\
& \left\|\int_{0}^{t} \widetilde{S}(s) v d s\right\| \leq \operatorname{Lim}_{n \rightarrow \infty} C\left(1-\frac{\omega t}{n}\right)^{-n}\|v\|=C e^{\omega t}\|v\|, \\
& \left\|B \int_{0}^{t} \widetilde{S}(s) v d s\right\| \leq C e^{\omega t}\|v\| \\
& \left\|B \int_{0}^{t}(t-s) \widetilde{S}(s) v d s\right\| \leq C e^{\omega t}\|v\|
\end{aligned}
$$

Accordingly, $\widetilde{S}(t),(t \geq 0)$ can be extended to all of $E$ as a bounded operator which we denote by the same symbol; recalling that $\widetilde{S}(0) v=0$ and $\widetilde{S}(t) v$ is continuous in $t \geq 0$ for $v \in D(A) \cap D(B)$, we can assert that $\widetilde{S}(0)=0$ and $\widetilde{S}(t)$ is strongly continuous in $t \geq 0$. By virtue of the denseness of $D(A) \cap D(B)$ and the uniform boundedness of

$$
\left.\frac{(-1)^{n-1}}{(n-1) !}\left(\frac{n}{t}\right)^{n}\left[\Delta(\lambda)^{-1}\right]^{(n-1)}\right|_{\lambda=n / t}
$$

on bounded subsets of $t>0$, we deduce that (2.5) is valid for all $v \in E$. Similarly, (2.6), (2.7) and (2.8) also hold for all $v \in E$ (here, the closedness of $B$ is used); moreover, $\int_{0}^{t} \widetilde{S}(s) v d s, B \int_{0}^{t} \widetilde{S}(s) v d s$ and $B \int_{0}^{t}(t-s) \widetilde{S}(s) v d s$ are continuous in $t \geq 0$ for $v \in E$.

Based on the paragraph above, we shall define several operators. First, define, for $t>0, u \in D(A)$,

(2.9) $\widetilde{C}(t) u=\operatorname{Lim}_{n \rightarrow \infty} \frac{(-1)^{n}}{(n-1) !}\left(\frac{n}{t}\right)^{n}\left\{\left[\frac{1}{\lambda} u\right]^{(n-1)}\right.$

$$
\begin{aligned}
& \left.-\left[\frac{1}{\lambda} \Delta(\lambda)^{-1} A u\right]^{(n-1)}\right\}\left.\right|_{\lambda=n / t} \\
& =u-\int_{0}^{t} \widetilde{S}(s) A u d s .
\end{aligned}
$$


Since for $u \in D(A) \cap D(B), \operatorname{Re} \lambda>\omega, n=0,1,2, \ldots$

$$
\begin{gathered}
\left\|\left[\frac{1}{\lambda} u-\frac{1}{\lambda} \Delta(\lambda)^{-1} A u\right]^{(n)}\right\| \\
=\left\|\left[\lambda \Delta(\lambda)^{-1} u+\Delta(\lambda)^{-1} B u\right]^{(n)}\right\| \\
\leq 2 C n !(\operatorname{Re} \lambda-\omega)^{-n-1}\|u\|,
\end{gathered}
$$

and $\Delta(\lambda)^{-1} A$ is closable, which implies that (2.10) is also valid for $u \in D(A)$, we have that for $u \in D(A), t>0$,

$$
\begin{aligned}
\|\widetilde{C}(t) u\| & \leq \operatorname{Lim}_{n \rightarrow \infty} 2 C \frac{1}{(n-1) !}\left(\frac{n}{t}\right)^{n}(n-1) !\left(\frac{n}{t}-\omega\right)^{-n}\|u\| \\
& =2 C e^{\omega t}\|u\|,
\end{aligned}
$$

thus $\widetilde{C}(t)(t>0)$ can be extended to a bounded operator on $E$ which we denote by the same symbol. Define $\widetilde{C}(0)=I$. Then $\widetilde{C}(t)$ is strongly continuous in $t \geq 0$.

Define, for $t>0, v \in D(A) \cap D(B)$,

$$
\begin{aligned}
\widetilde{K}(t) v & =\left.\operatorname{Lim}_{n \rightarrow \infty} \frac{(-1)^{n-1}}{(n-1) !}\left(\frac{n}{t}\right)^{n}\left[\lambda \Delta(\lambda)^{-1} v\right]^{(n-1)}\right|_{\lambda=n / t} \\
& =\operatorname{Lim}_{n \rightarrow \infty} \frac{(-1)^{n-1}}{(n-1) !}\left(\frac{n}{t}\right)^{n}\left\{\left[\frac{1}{\lambda} v\right]^{(n-1)}-\left[\Delta(\lambda)^{-1} B v\right]^{(n-1)}\right. \\
& \left.=v-\left[\frac{1}{\lambda} \Delta(\lambda)^{-1} A v\right]^{(n-1)}\right\}\left.\right|_{\lambda=n / t} \\
\widetilde{T}(t) v & =\left.\operatorname{Lim}_{n \rightarrow \infty} \frac{(-1)^{n-1}}{(n-1) !}\left(\frac{n}{t}\right)^{n}\left[B \Delta(\lambda)^{-1} v\right]^{(n-1)}\right|_{\lambda=n / t} ^{t} \widetilde{S}(s) A v d s, \quad-\left[\frac{1}{\lambda} B \Delta(\lambda)^{-1} B v\right] \\
& =\left.\operatorname{Lim}_{n \rightarrow \infty} \frac{(-1)^{n-1}}{(n-1) !}\left(\frac{n}{t}\right)^{n}\left\{\left[\frac{1}{\lambda^{2}} B v\right]^{(n-1)}-\left[\frac{1}{\lambda^{2}} B \Delta(\lambda)^{-1} A v\right]^{(n-1)}\right\}\right|_{\lambda=n / t} \\
& =-B \int_{0}^{t} \widetilde{S}(s) B v d s-B \int_{0}^{t}(t-s) \widetilde{S}(s) A v d s .
\end{aligned}
$$


Here the limits are uniform on compacts of $t>0$. But

$$
\begin{aligned}
\|K(t) v\| & =\left\|\left.\operatorname{Lim}_{n \rightarrow \infty} \frac{(-1)^{n-1}}{(n-1) !}\left(\frac{n}{t}\right)^{n}\left[\lambda \Delta(\lambda)^{-1} v\right]^{(n-1)}\right|_{\lambda=n / t}\right\| \\
& \leq \operatorname{Lim}_{n \rightarrow \infty} \frac{C}{(n-1) !}\left(\frac{n}{t}\right)^{n}(n-1) !\left(\frac{n}{t}-\omega\right)^{-n}\|v\|=C e^{\omega t}\|v\|, \\
\|\widetilde{T}(t) v\| & \leq \operatorname{Lim}_{n \rightarrow \infty} \frac{C}{(n-1) !}\left(\frac{n}{t}\right)^{n}(n-1) !\left(\frac{n}{t}-\omega\right)^{-n}\|v\| \\
& =C e^{\omega t}\|v\|
\end{aligned}
$$

therefore $\widetilde{K}(t)$ and $\widetilde{T}(t)(t>0)$ can be extended to all of $E$ as bounded operators which we denote by the same symbols. Define $\widetilde{K}(0)=I$, $\widetilde{T}(0)=0$, then $\widetilde{K}(t)$ and $\widetilde{T}(t)$ are strongly continuous in $t \geq 0$. Arguing as in the treatment of (2.5), we have that for all $v \in E$,

$$
\begin{aligned}
& \widetilde{K}(t) v=\left.\operatorname{Lim}_{n \rightarrow \infty} \frac{(-1)^{n-1}}{(n-1) !}\left(\frac{n}{t}\right)^{n}\left[\lambda \Delta(\lambda)^{-1} v\right]^{(n-1)}\right|_{\lambda=n / t}, \\
& \widetilde{T}(t) v=\left.\operatorname{Lim}_{n \rightarrow \infty} \frac{(-1)^{n-1}}{(n-1) !}\left(\frac{n}{t}\right)^{n}\left[B \Delta(\lambda)^{-1} v\right]\right|_{\lambda=n / t} .
\end{aligned}
$$

The limits are uniform on compacts of $t>0$.

Now, let us turn to (2.4). Define

$$
\begin{aligned}
& u(t ; u, v)=\operatorname{Lim}_{n \rightarrow \infty} U_{n}(t ; u, v) \\
& =\operatorname{Lim}_{n \rightarrow \infty} \frac{(-1)^{n-1}}{(n-1) !}\left(\frac{n}{t}\right)^{n}\left\{\left[\frac{1}{\lambda} u-\frac{1}{\lambda} \Delta(\lambda)^{-1} A u\right]^{(n-1)}\right. \\
& \left.+\left[\Delta(\lambda)^{-1} v\right]^{(n-1)}\right\}\left.\right|_{\lambda=n / t} \\
& =\widetilde{C}(t) u+\widetilde{S}(t) v \\
& v(t ; u, v)=\operatorname{Lim}_{n \rightarrow \infty} V_{n}(t ; u, v)=-\widetilde{S}(t) A u+\widetilde{K}(t) v
\end{aligned}
$$

where the limits are uniform on compacts of $t>0$. By the closedness 
of $A, B$, we obtain

$$
\begin{aligned}
& A U_{n}(t ; u, v)=\frac{(-1)^{n-1}}{(n-1) !}\left(\frac{n}{t}\right)^{n}\left\{\left[\frac{1}{\lambda} A u-\frac{1}{\lambda} A \Delta(\lambda)^{-1} A u\right]^{(n-1)}\right. \\
& \left.+\left[A \Delta(\lambda)^{-1} v\right]^{(n-1)}\right\}\left.\right|_{\lambda=n / t} \\
& =\frac{(-1)^{n-1}}{(n-1) !}\left(\frac{n}{t}\right)^{n}\left\{\left[\frac{1}{\lambda} A u-\frac{1}{\lambda} A \Delta(\lambda)^{-1} A u\right]^{(n-1)}\right. \\
& +\left[\frac{1}{\lambda^{2}} A v-\frac{1}{\lambda} A \Delta(\lambda)^{-1} B v\right. \\
& \left.\left.-\frac{1}{\lambda^{2}} A \Delta(\lambda)^{-1} A v\right]^{(n-1)}\right\}\left.\right|_{\lambda=n / t} \text {, } \\
& B V_{n}(t ; u, v)=\frac{(-1)^{n-1}}{(n-1) !}\left(\frac{n}{t}\right)^{n}\left\{\left[-B \Delta(\lambda)^{-1} A u\right]^{(n-1)}\right. \\
& \left.+\left[\lambda B \Delta(\lambda)^{-1} v\right]^{(n-1)}\right\}\left.\right|_{\lambda=n / t} \\
& =\frac{(-1)^{n-1}}{(n-1) !}\left(\frac{n}{t}\right)^{n}\left\{\left[-B \Delta(\lambda)^{-1} A u\right]^{(n-1)}\right. \\
& +\left[\frac{1}{\lambda} B v-B \Delta(\lambda)^{-1} B v\right. \\
& \left.\left.-\frac{1}{\lambda} B \Delta(\lambda)^{-1} A v\right]^{(n-1)}\right\}\left.\right|_{\lambda_{n / t}} \text {. }
\end{aligned}
$$

Also

$$
\frac{1}{\lambda} A \Delta(\lambda)^{-1}=\frac{1}{\lambda} I-B \Delta(\lambda)^{-1}-\lambda \Delta(\lambda)^{-1}
$$

therefore we have

(2.15) $A u(t ; u, v)=\operatorname{Lim}_{n \rightarrow \infty} A U_{n}(t ; u, v)$

$$
\begin{aligned}
= & \widetilde{T}(t) A u+\widetilde{K}(t) A u-B v+\widetilde{T}(t) B v+\widetilde{K}(t) B v \\
& +B \int_{0}^{t} \widetilde{S}(s) A v d s+\widetilde{S}(t) A v,
\end{aligned}
$$

(2.16) $B v(t ; u, v)=\operatorname{Lim}_{n \rightarrow \infty} B V_{n}(t ; u, v)$

$$
=-\widetilde{T}(t) A u+B v-\widetilde{T}(t) B v-B \int_{0}^{t} \widetilde{S}(s) A v d s,
$$


where the limits are uniform on compacts of $t>0$. Accordingly, as $n \rightarrow \infty$, (2.4) becomes

$$
\left\{\begin{array}{l}
\frac{d}{d t} u(t ; u, v)=v(t ; u, v), \\
\frac{d}{d t} v(t ; u, v)=-B v(t ; u, v)-A u(t ; u, v),
\end{array}\right.
$$

in $t>0$. Define $u(0 ; u, v)=u, v(0 ; u, v)=v$ for $u \in D(A), v \in D(A) \cap$ $D(B)$; then by (2.13), (2.14), (2.15) and (2.16), $u(t ; u, v), v(t ; u, v)$, $B v(t ; u, v)$ and $A u(t ; u, v)$ are continuous in $t \geq 0$, and therefore (2.17) holds in $t \geq 0$, i.e.

$$
u^{\prime \prime}(t ; u, v)+B u^{\prime}(t ; u, v)+A u(t ; u, v)=0 \quad(t \geq 0) .
$$

We have then proved that the equation (1.1) has a solution for every initial value $\left(u(0), u^{\prime}(0)\right) \in(D(A) \cap D(B))^{2}$. It remains to show continuous dependence on initial data, in view of the fact that the statement (ii) implies the statement (i) (as proved before). To this end we observe that, from (2.9) and combining with (2.11), (2.13), (2.14) and (2.17), we obtain

$$
\begin{aligned}
\widetilde{C}(t) u & =\left.\operatorname{Lim}_{n \rightarrow \infty} \frac{(-1)^{n-1}}{(n-1) !}\left(\frac{n}{t}\right)^{n}\left[\Delta(\lambda)^{-1}(\lambda+B) u\right]^{(n-1)}\right|_{\lambda=n / t} \\
& =\widetilde{S}^{\prime}(t) u+\widetilde{S}(t) B u
\end{aligned}
$$

for $u \in D(A) \cap D(B), t>0$. Hence,

$$
\text { (2.19) } \widetilde{S}(t) u=\int_{0}^{t}[\widetilde{C}(s) u-\widetilde{S}(s) B u] d s \quad(u \in D(A) \cap D(B), t \geq 0) \text {. }
$$

Let now $w(t)$ be an arbitrary solution of (1.1). Set, for $t \geq 0$, ( $n$ a natural number), $\varphi_{n}(t)=\int_{0}^{1 / n} n(s+1) w^{\prime}(t+s) d s$. It is clear that for $t \geq 0, \varphi_{n}(t) \in D(B), \varphi_{n}(t) \rightarrow w^{\prime}(t)$ and $B \varphi_{n}(t) \rightarrow B w^{\prime}(t)$ as $n \rightarrow \infty$. Moreover, integrating by parts, we obtain

$$
\varphi_{n}(t)=\left.n(s+1) w(t+s)\right|_{0} ^{1 / n}-\int_{0}^{1 / n} n w(s+t) d s \in D(A) \quad(t \geq 0) .
$$

Thus (2.19) holds for $u \in w^{\prime}(t)(t \geq 0)$. From this and (2.9), we deduce

$$
\begin{aligned}
\frac{d}{d s}[\widetilde{C} & \left.(t-s) w(s)+\widetilde{S}(t-s) w^{\prime}(s)\right] \\
= & -\widetilde{C}^{\prime}(t-s) w(s)+\widetilde{C}(t-s) w^{\prime}(s)-\widetilde{S}^{\prime}(t-s) w^{\prime}(s) \\
& +\widetilde{S}(t-s) w^{\prime \prime}(s) \\
= & \widetilde{S}(t-s) A w(s)+\widetilde{C}(t-s) w^{\prime}(s)-\widetilde{C}(t-s) w^{\prime}(s) \\
& +\widetilde{S}(t-s) B w^{\prime}(s)+\widetilde{S}(t-s)\left[-B w^{\prime}(s)-A w(s)\right] \\
= & 0 \quad(0 \leq s \leq t)
\end{aligned}
$$


Consequently

$$
w(t)=\widetilde{C}(t) w(0)+\widetilde{S}(t) w^{\prime}(0) \quad(t \geq 0),
$$

and this ends the proof of the implication (iii) $\Rightarrow(\mathrm{i})$.

Finally, we show the explicit expressions (1.4) to (1.9). By virtue of the equivalence of (2.1) and (2.2) in Lemma 1, (1.4) (resp. (1.5)) results from (2.9) (resp. (2.5)). Recalling that for $\operatorname{Re} \lambda>\omega$,

$$
\begin{aligned}
\Delta(\lambda)^{-1} u & =\int_{0}^{\infty} e^{-\lambda t} S(t) u d t, \\
\lambda \Delta(\lambda)^{-1} u & =\int_{0}^{\infty} e^{-\lambda t} S^{\prime}(t) u d t \quad(u \in E), \\
\frac{1}{\lambda} \Delta(\lambda)^{-1} A u & =\int_{0}^{\infty} e^{-\lambda t}\left[\int_{0}^{t} S(s) A u d s\right] \quad(u \in D(A)),
\end{aligned}
$$

then (1.6) follows from (2.9) and Lemma 2, (1.7) from Lemma 2, (1.8) from (2.9) and Lemma 3, (1.9) from Lemma 3. Thus we have completed the proof of Theorem 1 .

3. Applications of Theorem 1. F. Neubrander [5] has discussed the case $D(A) \supset D(B)$. He shows well posedness under the assumption that $-B$ is the generator of a strongly continuous semigroup and $R(\lambda,-B) A=A R(\lambda,-B)$ on $D(A)$ for $\operatorname{Re} \lambda=\omega(\omega$ a constant $)$. As a consequence of Theorem 1 , the following theorem generalizes the result.

THEOREM 2. Suppose $D(A) \supset D(B)$ and there exists a complex number $\lambda_{0}$ such that $\left(\lambda_{0} I+B\right)^{-1} A$ has bounded extension. Then the Cauchy problem for (1.1) is strongly well posed if and only if $-B$ is the generator of a strongly continuous semigroup.

Proof. Necessity. According to [1, Ch. VIII, Corollary 3.5], for every initial value $\left(u_{0}, u_{1}\right) \in D(B) \times D(B)$ the Cauchy problem for (1.1) has a solution. This ends the proof by the well posedness and [5, Theorem 5].

Sufficiency. We assume $\lambda_{0}=0$ without the loss of generality. Let now $(T(t))$ be the semigroup generated by $-B$. It is well known that there exist $C, \omega \geq 0$ such that

$$
\begin{gathered}
(\lambda I+B)^{-1} u=\int_{0}^{\infty} e^{-\lambda t} T(t) u d t \quad(u \in E, \operatorname{Re} \lambda>\omega), \\
\|T(t)\| \leq C e^{\omega t} \quad(t \geq 0) .
\end{gathered}
$$


Hence, we have

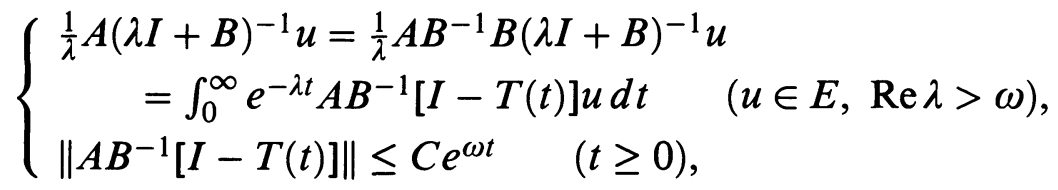

(here and in the sequel, we denote by $C$ a generic constant).

It is clear that for $\operatorname{Re} \lambda>\omega+C$,

$$
\begin{aligned}
\lambda \Delta(\lambda)^{-1} & =(\lambda I+B)^{-1}\left[I+\frac{1}{\lambda} A(\lambda I+B)^{-1}\right]^{-1} \\
& =(\lambda I+B)^{-1} \sum_{n=0}^{\infty}\left[-\frac{1}{\lambda} A(\lambda I+B)^{-1}\right]^{n} .
\end{aligned}
$$

In order to estimate $\left[\lambda \Delta(\lambda)^{-1}\right]^{(n)}$ for $n=0,1,2 \ldots$, we observe that, by (3.1),

$\left[(\lambda I+B)^{-1} u\right]^{(m)}=\int_{0}^{\infty}(-t)^{m} e^{-\lambda t} T(t) u d t \quad(u \in E, m=0,1,2, \ldots)$, and therefore

$$
\begin{aligned}
& \left.\|(\lambda I+B)^{-1}\right]^{(m)} \| \leq \int_{0}^{\infty} t^{m} e^{-\operatorname{Re} \lambda t} C e^{\omega t} d t \\
& \quad=C m !(\operatorname{Re} \lambda-\omega)^{-m-1} \quad(m=0,1,2, \ldots) .
\end{aligned}
$$

Set $Q(t)=A B^{-1}[I-T(t)]$ for $t \geq 0$. Then by (3.2),

$$
\left[\frac{1}{\lambda} A(\lambda I+B)^{-1}\right]^{n} u=\int_{0}^{\infty} e^{-\lambda t}[Q(t)]^{* n} u d t \quad(u \in E, n=2,3, \ldots),
$$

where $* n$ indicates the $n$th convolution power. Consequently, we obtain

$$
\begin{aligned}
\left\{\left[\frac{1}{\lambda} A(\lambda I+B)^{-1}\right]^{n} u\right\}^{(m)}=\int_{0}^{\infty}(-t)^{m} e^{-\lambda t}[Q(t)]^{* n} u d t \\
\\
(u \in E, m=1,2, \ldots, n=2,3, \ldots) .
\end{aligned}
$$

But for $u \in E, t \geq 0, n=2,3, \ldots$,

$$
\begin{array}{r}
{[Q(t)]^{* n} u=\int_{0}^{t} \int_{0}^{t_{n-2}} \cdots \int_{0}^{t_{1}} Q\left(t-t_{n-2}\right) Q\left(t_{n-2}-t_{n-3}\right)} \\
\cdots Q\left(t_{1}-t_{0}\right) Q\left(t_{0}\right) u d t_{0} d t_{1} \cdots d t_{n-2}
\end{array}
$$

so that,

$$
\begin{aligned}
\left\|[Q(t)]^{* n}\right\| & \leq \int_{0}^{t} \int_{0}^{t_{n-2}} \cdots \int_{0}^{t_{1}} C^{n} e^{\omega t} d t_{0} \cdots d t_{n-2} \\
& =C^{n} e^{\omega t} \frac{t^{n-1}}{(n-1) !}
\end{aligned}
$$


hence for $m=1,2, \ldots, n=2,3, \ldots$,

$$
\begin{gathered}
\left\|\left\{\left[\frac{1}{\lambda} A(\lambda I+B)^{-1}\right]^{n}\right\}^{(m)}\right\| \leq \int_{0}^{\infty} t^{m} e^{-\operatorname{Re} \lambda t} C^{n} e^{\omega t} \frac{t^{n-1}}{(n-1) !} d t \\
=\frac{C^{n}}{(n-1) !}(m+n-1) !(\operatorname{Re} \lambda-\omega)^{-m-n} .
\end{gathered}
$$

When $n=1$, making use of (3.2) again, we obtain easily that for $m=0,1,2, \ldots$,

$$
\begin{aligned}
\left\|\left[\frac{1}{\lambda} A(\lambda I+B)^{-1}\right]^{(m)}\right\| & \leq \int_{0}^{\infty} t^{m} e^{-\operatorname{Re} \lambda t} C e^{\omega t} d t \\
& =C m !(\operatorname{Re} \lambda-\omega)^{-m-1} .
\end{aligned}
$$

Accordingly, we have

$$
\begin{aligned}
& \left\|\left\{\sum_{n=0}^{\infty}\left[-\frac{1}{\lambda} A(\lambda I+B)^{-1}\right]^{n}\right\}^{(m)}\right\| \\
& \leq \sum_{n=1}^{\infty} \frac{C^{n}}{(n-1) !}(m+n-1) !(\operatorname{Re} \lambda-\omega)^{-m-n} \\
& =\frac{C}{(\operatorname{Re} \lambda-\omega)^{m+1}} \sum_{n=0}^{\infty}\left[\left(\frac{C}{\operatorname{Re} \lambda-\omega}\right)^{n}(n+1)(n+2) \cdots(m+n)\right] \\
& =\left.\frac{C}{(\operatorname{Re} \lambda-\omega)^{m+1}}\left(\frac{d^{m}}{d x^{m}} \sum_{n=0}^{\infty} x^{m+n}\right)\right|_{x=C / \operatorname{Re} \lambda-\omega} \\
& =C m !(\operatorname{Re} \lambda-\omega-C)^{-(m+1)} \quad(m=1,2, \ldots),
\end{aligned}
$$

$$
\begin{aligned}
& \left\|\sum_{n=0}^{\infty}\left[-\frac{1}{\lambda} A(\lambda I+B)^{-1}\right]^{n}\right\| \\
& \quad \leq \frac{1}{1-C(\operatorname{Re} \lambda-\omega)^{-1}}=\frac{\operatorname{Re} \lambda-\omega}{\operatorname{Re} \lambda-\omega-C} \quad(m=0) .
\end{aligned}
$$

We now apply the Leibniz formula to (3.3), obtaining

$$
\begin{aligned}
{\left[\lambda \Delta(\lambda)^{-1}\right]^{(n)}=} & \sum_{k=0}^{n} C_{n}^{k}\left[(\lambda I+B)^{-1}\right]^{(k)} \\
& \cdot\left\{\sum_{m=0}^{\infty}\left[-\frac{1}{\lambda} A(\lambda I+B)^{-1}\right]^{m}\right\}^{(n-k)} \quad(n=0,1,2, \ldots) .
\end{aligned}
$$


It is thus easy to see, using (3.4), (3.5) and (3.6), that

$$
\begin{aligned}
&\left\|\left[\lambda \Delta(\lambda)^{-1}\right]^{(n)}\right\| \\
& \leq \sum_{k=0}^{n-1} C_{n}^{k} C k !(\operatorname{Re} \lambda-\omega)^{-k-1} C(n-k) !(\operatorname{Re} \lambda-\omega-C)^{-n+k-1} \\
&+C n !(\operatorname{Re} \lambda-\omega)^{-n-1}(\operatorname{Re} \lambda-\omega)(\operatorname{Re} \lambda-\omega-C)^{-1} \\
&= C^{2} n !(\operatorname{Re} \lambda-\omega-C)^{-n-1}(\operatorname{Re} \lambda-\omega)^{-1} \\
& \times\left\{\sum_{k=0}^{n-1}\left(\frac{\operatorname{Re} \lambda-\omega-C}{\operatorname{Re} \lambda-\omega}\right)^{k}+\sum_{k=n}^{\infty}\left(\frac{\operatorname{Re} \lambda-\omega-C}{\operatorname{Re} \lambda-\omega}\right)^{k}\right\} \\
&= C n !(\operatorname{Re} \lambda-\omega-C)^{-n-1} \quad(\operatorname{Re} \lambda>\omega+C, n=0,1,2, \ldots) .
\end{aligned}
$$

Similarly, we can obtain the other two estimations:

$$
\begin{array}{r}
\left\|\left[B \Delta(\lambda)^{-1}\right]^{(n)}\right\| \leq C n !(\operatorname{Re} \lambda-\omega-C)^{-n-1} \\
(\operatorname{Re} \lambda>\omega+C, n=0,1,2, \ldots)
\end{array}
$$

and from

$$
\begin{array}{r}
\Delta(\lambda)^{-1} B u=\left(I+\frac{1}{\lambda} B(\lambda I+B)^{-1} \overline{B^{-1} A}\right)^{-1} \frac{1}{\lambda}(\lambda I+B)^{-1} B u \\
(u \in D(B), \operatorname{Re} \lambda>\omega+C),
\end{array}
$$

we obtain

$$
\begin{aligned}
\left\|\left[\Delta(\lambda)^{-1} B u\right]^{(n)}\right\| \leq C n ! & (\operatorname{Re} \lambda-\omega-C)^{-n-1} \\
& (u \in D(B), \operatorname{Re} \lambda>\omega+C, n=0,1,2, \ldots) .
\end{aligned}
$$

According to Theorem 1, the proof is now complete.

On the other hand, we discuss the case where $D(A) \subset D(B)$. We have shown in the proof of the implication (ii) $\Rightarrow$ (i) in Section 2 that the general case can, in a certain way, be reduced to this one. The case where $B \in L(E)$ has been studied in [12], and the conclusion is that the Cauchy problem for (1.1) is well posed (or equivalently, in view of [2, Th. 4.1(a)], strongly well posed) if and only if $-A$ is the generator of a strongly continuous cosine function. As an example of the case when $D(B) \supset D(A)$ and $B$ is unbounded, we now investigate 
the partial differential equation

$$
\left\{\begin{array}{l}
\frac{\partial^{2} u(x, t)}{\partial t^{2}}+\frac{\partial}{\partial x}\left(a(x) \frac{\partial}{\partial x} u(x, t)\right) \\
+c \frac{\partial^{4}}{\partial x^{4}} u(x, t)+i b \frac{\partial^{3}}{\partial t \partial x^{2}} u(x, t)=0, \\
\left.u(x, t)\right|_{x=0,1}=\left.\frac{\partial^{2}}{\partial x^{2}} u(x, t)\right|_{x=0,1}=0 \quad(t \geq 0), \\
u(x, 0)=u_{0}(x), \quad \frac{\partial}{\partial t} u(x, 0)=u_{1}(x) \quad(0 \leq x \leq 1) .
\end{array}\right.
$$

Let $E=L^{2}(0,1), a(x) \in C^{1}(0,1), c>0$ and $b$ be a real number; $A=A_{1}+A_{2}$, where

$$
A_{1}=\frac{\partial}{\partial x}\left(a(x) \frac{\partial}{\partial x} \cdot\right)
$$

with

$$
D\left(A_{1}\right)=\left\{u \in H^{2}(0,1),\left.u(x)\right|_{x=0,1}=0\right\}
$$

and

$$
\begin{aligned}
A_{2} & =c \frac{\partial^{4}}{\partial x^{4}} \quad \text { with } \\
D\left(A_{2}\right) & =\left\{u \in H^{4}(0,1),\left.u(x)\right|_{x=0,1}=\left.u^{\prime \prime}(x)\right|_{x=0,1}=0\right\},
\end{aligned}
$$

and

$$
\begin{gathered}
B=i b \frac{\partial^{2}}{\partial x^{2}} \quad \text { with } D(B)=D\left(A_{1}\right) \\
B_{1}=c^{1 / 2} \frac{\partial^{2}}{\partial x^{2}} \quad \text { with } D\left(B_{1}\right)=D(B)
\end{gathered}
$$

so that $B_{1}^{2}=A_{2}$. Then it is easy to see that $A$ and $B$ are all closed operators, $B_{1} B=B B_{1}, B_{1}^{*}=B_{1}, B^{*}=-B, A_{1} B^{-1} \in L(E)$ and $B^{-1} A_{1}$ has a bounded extension due to the fact that $B^{-1} A_{1} \subset\left(A_{3} B^{-1}\right)^{*}$ and $A_{3} B^{-1} \in L(E)$ where

$$
A_{3}=-\frac{\partial}{\partial x}\left(\overline{a(x)} \frac{\partial}{\partial x}\right)
$$

with $D\left(A_{3}\right)=D(B)$. Set

$$
F=\left(\begin{array}{cc}
0 & B_{1} \\
-B_{1} & B
\end{array}\right) \quad \text { with } D(F)=D(B) \times D(B) \text { in } E \times E .
$$


Then $(i F)^{*}=i F$, thus it follows from [7, P. 41, Th. 10.8] that $F$ is the generator of a strongly continuous unitary group. Denote this group by

$$
\left(\begin{array}{ll}
T_{1}(t) & T_{2}(t) \\
T_{3}(t) & T_{4}(t)
\end{array}\right) \quad \text { for }-\infty<t<+\infty
$$

Observing that

$$
R(\lambda, F)=\left(\begin{array}{cc}
(\lambda I+B) \Delta_{1}(\lambda)^{-1} & B_{1} \Delta_{1}(\lambda)^{-1} \\
B_{1} \Delta_{1}(\lambda)^{-1} & \lambda \Delta_{1}(\lambda)^{-1}
\end{array}\right) \quad \text { for } \operatorname{Re} \lambda>0
$$

where $\Delta_{1}(\lambda)=\lambda^{2} I+\lambda B+A_{2}$, we obtain that for $\operatorname{Re} \lambda>0$,

$$
\left\{\begin{array}{c}
\lambda \Delta_{1}(\lambda)^{-1} u=\int_{0}^{\infty} e^{-\lambda t} T_{4}(t) u d t \quad(u \in E) \\
\left\|T_{4}(t)\right\| \leq 1(t \geq 0), \\
B \Delta_{1}(\lambda)^{-1} u=\int_{0}^{\infty} e^{-\lambda t}\left[T_{1}(t)-T_{4}(t)\right] u d t \quad(u \in E), \\
\left\|T_{1}(t)-T_{4}(t)\right\| \leq 2(t \geq 0) .
\end{array}\right.
$$

Therefore

$$
\left\{\begin{array}{l}
A_{1} \Delta_{1}(\lambda)^{-1} u=A_{1} B^{-1} B \Delta_{1}(\lambda)^{-1} u \\
\quad=\int_{0}^{\infty} e^{-\lambda t} A_{1} B^{-1}\left[T_{1}(t)-T_{4}(t)\right] u d t \quad(u \in E), \\
\left\|A_{1} B^{-1}\left[T_{1}(t)-T_{4}(t)\right]\right\| \leq 2\left\|A_{1} B^{-1}\right\| \quad(t \geq 0)
\end{array}\right.
$$

(3.10) $\overline{\Delta_{1}(\lambda)^{-1}} A_{1} u=B \Delta_{1}(\lambda)^{-1} \overline{B^{-1} A_{1}} u$

$$
\begin{aligned}
& =\int_{0}^{\infty} e^{-\lambda t}\left[T_{1}(t)-T_{4}(t)\right] \overline{B^{-1} A_{1}} u d t \quad(u \in E), \\
\|\left[T_{1}(t)\right. & \left.-T_{4}(t)\right] \overline{B^{-1} A_{1}}\|\leq 2\| \overline{B^{-1} A_{1}} \| \quad(t \geq 0) .
\end{aligned}
$$

From (3.8), (3.9), (3.10) and the plain fact that

$$
\begin{aligned}
\lambda \Delta(\lambda)^{-1} & =\lambda \Delta_{1}(\lambda)^{-1}\left[I+A_{1} \Delta_{1}(\lambda)^{-1}\right]^{-1} & & (\operatorname{Re} \lambda>0), \\
B \Delta(\lambda)^{-1} & =B \Delta_{1}(\lambda)^{-1}\left[I+A_{1} \Delta_{1}(\lambda)^{-1}\right] & & (\operatorname{Re} \lambda>0), \\
\Delta(\lambda)^{-1} B u & =\left[I+\overline{\Delta_{1}(\lambda)^{-1}} A_{1}\right]^{-1} B \Delta_{1}(\lambda)^{-1} u & & (u \in E, \operatorname{Re} \lambda>0),
\end{aligned}
$$

we can obtain the three estimations in (iii) of Theorem 1 using arguments similar to those in the proof of the sufficiency of Theorem 2. Then, applying Theorem 1, we conclude that (3.7) is strongly well posed and therefore the propagators grow exponentially.

Acknowledgment. We are grateful to our advisor Professor F. L. Huang for his generous guidance. 


\section{REFERENCES}

[1] H. O. Fattorini, Second Order Linear Differential Equations in Banach Spaces, (1985), Elsevier Science Publishers B. V., Amsterdam.

[2] _ Extension and behavior at infinity of solutions of certain linear operational differential equations, Pacific J. Math., 33 (1970), 583-615.

[3] E. Hewitt, Real and Abstract Analysis, (1965), Springer-Verlag, Berlin.

[4] E. Hille and R. S. Phillips, Functional analysis and semigroups, (1957), Amer. Math. Soc. Colloquium Pubs. vol. 31, Providence.

[5] F. Neubrander, Well-posedness of higher order abstract Cauchy problems, Trans. Amer. Math. Soc., 295 (1986), 257-290.

[6] _ On the relation between the semigroup and its infinitesimal generator, Proc. Amer. Math. Soc., 100 (1987), 104-107.

[7] A. Pazy, Semigroups of Linear Operators and Applications to Partial Differential Equations, (1983), Springer-Verlag, New York and Berlin.

[8] G. Da Prato and E. Giusti, Una caratterizzazione dei generatori di funzioni coseno astratte. Boll. Un. Mat. Italiana (3), 22 (1967), 357-362.

[9] M. Sova, Cosine operator functions, Rozprawy Mat., 49 (1966), 1-47.

[10] __ Problème de Cauchy pour équations hyperboliques opérationnelles a coefficients constants non-bornés, Ann. Scuola Norm. Sup. Pisa, 22 (1968), 67-100.

[11] D. V. Widder, The Laplace transform, (1946), Princeton University Press, Princeton.

[12] T. J. Xiao, On the well-posedness of the Cauchy problem for a kind of complete second order linear differential equation, Sichuan University Natural Sci. Edition (Chinese) 25, No. 4 (1988), 421-428.

Received December 28, 1987 and in revised form, March 13, 1989.

YunNan Teachers UNIVERSITY

Kunming, People's Republic of China

AND

KUNMING ENGINEERING INSTITUTE

Kunming, People's Republic of China 



\section{PACIFIC JOURNAL OF MATHEMATICS EDITORS}

\author{
V. S. VARADARAJAN \\ (Managing Editor) \\ University of California \\ Los Angeles, CA 90024-1555-05 \\ Herbert Clemens \\ University of Utah \\ Salt Lake City, UT 84112 \\ ThOMAS ENRIGHT \\ University of California, San Diego \\ La Jolla, CA 92093
}

\section{R. FINN}

Stanford University

Stanford, CA 94305

HeRmann FlaschKa

University of Arizona

Tucson, AZ 85721

VAUGHAN F. R. JONES

University of California

Berkeley, CA 94720

Steven KerckhofF

Stanford University

Stanford, CA 94305

\author{
RobION KIRBY \\ University of California \\ Berkeley, CA 94720 \\ C. C. Moore \\ University of California \\ Berkeley, CA 94720 \\ HAROLD STARK \\ University of California, San Diego \\ La Jolla, CA 92093
}

\section{ASSOCIATE EDITORS}
R. ARENS
E. F. BECKenBach
B. H. NeumanN
F. WOLF
K. YoshidA (1906-1982)
(1904-1989)

\section{SUPPORTING INSTITUTIONS}

UNIVERSITY OF ARIZONA

UNIVERSITY OF BRITISH COLUMBIA

CALIFORNIA INSTITUTE OF TECHNOLOGY

UNIVERSITY OF CALIFORNIA

MONTANA STATE UNIVERSITY

UNIVERSITY OF NEVADA, RENO

NEW MEXICO STATE UNIVERSITY

OREGON STATE UNIVERSITY
UNIVERSITY OF OREGON

UNIVERSITY OF SOUTHERN CALIFORNIA

STANFORD UNIVERSITY

UNIVERSITY OF HAWAII

UNIVERSITY OF TOKYO

UNIVERSITY OF UTAH

WASHINGTON STATE UNIVERSITY

UNIVERSITY OF WASHINGTON 


\section{Pacific Journal of Mathematics}

Vol. 142, No. $1 \quad$ January, 1990

Marco Andreatta, Mauro Beltrametti and Andrew Sommese, Generic properties of the adjuction mapping for singular surfaces and applications

Chen-Lian Chuang and Pjek-Hwee Lee, On regular subdirect products of

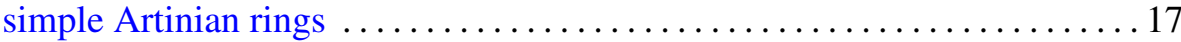

Fernando Giménez and Vicente Miquel Molina, Volume estimates for real hypersurfaces of a Kaehler manifold with strictly positive holomorphic sectional and antiholomorphic Ricci curvatures $\ldots \ldots \ldots \ldots \ldots \ldots 23$

Richard J. Griego and Andrzej Korzeniowski, Asymptotics for certain Wiener integrals associated with higher order differential operators

Abdeslam Mesnaoui, Unitary bordism of classifying spaces of quaternion

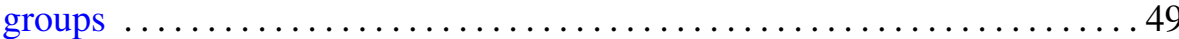

Abdeslam Mesnaoui, Unitary cobordism of classifying spaces of quaternion

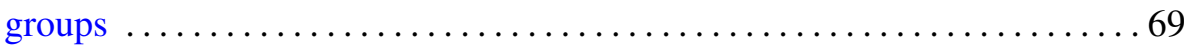

Jesper M. Møller, On equivariant function spaces $\ldots \ldots \ldots \ldots \ldots \ldots \ldots \ldots$

Bassam Nassrallah, A $q$-analogue of Appell's $F_{1}$ function, its integral representation and transformations

Peter A Ohring, Solvability of invariant differential operators on metabelian

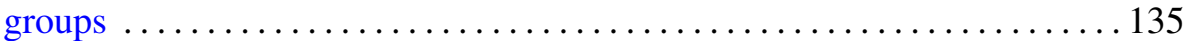

Athanase Papadopoulos and R. C. Penner, Enumerating pseudo-Anosov foliations

Ti-Jun Xiao and Liang Jin, On complete second order linear differential

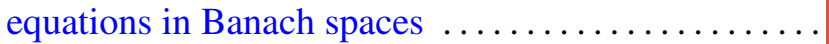

Carl Widland and Robert F. Lax, Weierstrass points on Gorenstein curves 